\title{
Cool dwarfs stars from the Torino Observatory Parallax Program
}

\author{
R. L. Smart ${ }^{1}$, G. Ioannidis ${ }^{2}$, H. R. A. Jones ${ }^{2}$, B. Bucciarelli ${ }^{1}$, and M. G. Lattanzi ${ }^{1}$ \\ 1 INAF/Osservatorio Astronomico di Torino, Strada Osservatorio 20, 10025 Pino Torinese, Italy \\ e-mail: smart@oato.inaf.it \\ 2 Centre for Astrophysics Research, Science and Technology Research Institute, University of Hertfordshire, \\ Hatfield AL10 9AB, UK
}

Received 8 October 2009 / Accepted 2 March 2010

ABSTRACT

\begin{abstract}
Aims. We investigate and parameterize high proper motion red stars in the Torino Observatory Parallax Program.
Methods. Observations of 27 objects were made over the period 1994-2001 on the $1.05 \mathrm{~m}$ Torino telescope. The trigonometric parallaxes and proper motions were determined using standard techniques.

Results. We determine parallaxes and proper motions, and by comparison to models we infer masses, ages, and metallicities. Of the 27 objects, 22 are within $25 \mathrm{pc}$ and 4 appear to be subdwarfs. There are published parallaxes for 18 objects, and all but 4 agree to within $2 \sigma$. The discrepancies are discussed.
\end{abstract}

Key words. astrometry - stars: low-mass - stars: fundamental parameters - stars: distances Hertzsprung-Russell (HR) and C-M diagrams

\section{Introduction}

Stars of spectral type M make up over $70 \%$ of the stars and $40 \%$ of the mass in our Galaxy. Their main sequence lifetimes can exceed the age of the universe, and they have age indicators that can be calibrated (e.g. Gizis et al. 2002) to make them useful chronometers. A wide variety of problems in Astronomy from the search for earth-like exoplanets (Torres 2007) to modeling the Galaxy (Jurić et al. 2008) are addressed using observations of these objects.

Notwithstanding their numerical dominance and potential scientific use, M stars make a surprising small proportion of objects that have a measured parallax. In the combined Hipparcos (Perryman et al. 1997) Yale Trigonometric Parallax (van Altena et al. 2001) and RECONS (Research Consortium on Nearby Stars, Henry et al. 2004) catalogues there are over 120000 stars of which less than 2000 are M dwarfs. In Fig. 1 we plot the number of M dwarfs with parallaxes as a function of spectral class. Approximately one half have relative parallax errors of less than $10 \%$ as shown by the shaded area. For the types later than M5 the total number per bin is always less than 30 . This is primarily because they are intrinsically low-luminosity objects; of the 40000 stars with apparent magnitude $V<8.0$ in the Hipparcos catalogue there are only four M dwarfs.

The majority of the late $\mathrm{M}$ dwarfs that have measured parallaxes have been determined using CCD observations, e.g. USNO (Monet et al. 1992) and RECONS programs. Here we present the M dwarfs found in the Torino Observatory Parallax Program (Smart et al. 2003, hereafter TOPP) and we use recent models to estimate mass and age for these objects. In Sect. 2 we present the TOPP observations and reduction procedures, in Sect. 3 we present the resulting parallaxes and proper motions, in Sect. 4 we determine mass and age, and in Sect. 5 we discuss individual objects.

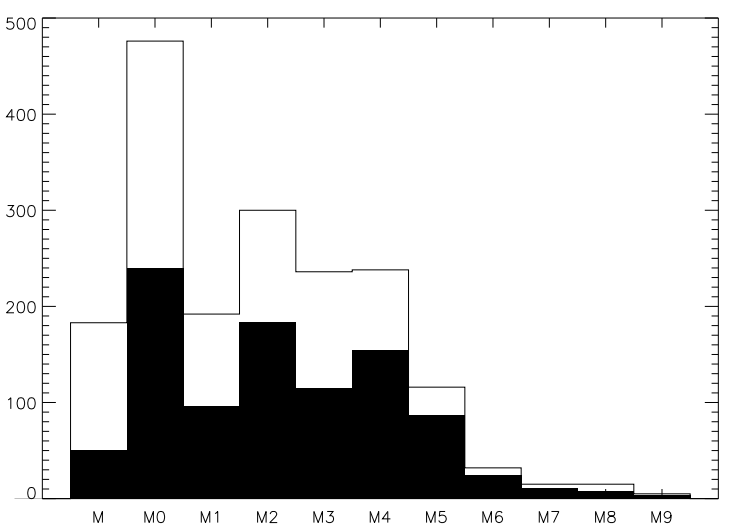

Fig. 1. M dwarfs with parallaxes as a function of subclass from a combination of the Hipparcos Yale Trigonometric Parallax and RECONS catalogues. The filled-in area represents those objects with errors of less than $10 \%$.

\section{Observations and reduction procedures}

The procedures for observation, image treatment and parallax determination follow those described in previous papers (Smart et al. 1999, 2003). These objects are very red compared to the field stars so a correction for differential colour refraction as presented in Smart et al. (2007) is applied. Here we outline the major steps of data reduction and the reader is referred to those publications for further details.

Observations were all carried out on the Torino Observatory $1.05 \mathrm{~m}$ reflecting telescope which is a scaled-down version of the $1.55 \mathrm{~m}$ Kaj Strand Astrometric Reflector at the USNO Flagstaff Station (Strand 1966). The CCD used was an EEV CCD05-30 1296×1152@15 microns/pixel constructed by the Astromed 
company which provided a pixel scale of $0.47^{\prime \prime}$ and a field of view of $10^{\prime} \times 9^{\prime}$. All parallax observations were carried out in the Cousins $I$ filter.

The images are flat-fielded using sky flats taken each night and bias-corrected using the overscan region of the CCD. All objects are found on each frame and centered using the Robin software (Lanteri 1990). A base frame is chosen with the criteria that it was made in good seeing and in the middle of the parallax observational sequence. Anonymous reference stars are selected automatically following two criteria: they are on at least $80 \%$ of the frames, the residual between predicted and observed coordinates is less than three times the overall mean residual. Selection of frames is also carried out automatically eliminating any frames that have a small number of stars in common with the base frame $(<9)$ or positional residuals larger than three times the average frame residual.

A given sequence is iterated to obtain proper motions and parallaxes for all objects and the above criteria are applied until the parallax of the target changes by less than $1 \%$. From the target star's relative parallax we must subtract the mean parallax of the reference stars as we have implicitly assumed that the reference frame is at a mean parallax of zero. Using the Mendez \& van Altena (1996) galaxy model we can calculate the most probable distance of each reference star based on their magnitude. The mean of these distances is an estimate of the correction to absolute parallax that we add to the target's relative parallax (COR, in Table 1). Relative parallax errors are found from the formal scatter about the final fit; to this we add, in quadrature, $30 \%$ of the relative to absolute correction which we estimate to be the error of the galactic model procedure used (Smart et al. 1997).

We note that all these targets were solved assuming a simple single star solution; an extension to double star solutions will be made for those stars with suspect or visual companions. For the visual binaries in this study the orbital period is of the order of centuries so the difference will be negligible. For the nonresolved binaries we still need to develop appropriate software and routines; any effect, if present, will be reflected in the errors (e.g. LHS 1976 discussed in Sect. 5).

\section{Results}

In Table 1 we report the TOPP results for the 27 red dwarfs. These stars were originally selected for a number of reasons: 4 were chosen to overlap with the USNO program, 4 were part of the TOPP investigation into the catalogue of nearby stars (Smart et al. 2007) and the rest are visual binary systems and high proper motions stars, historically subjects of interest at the Torino Observatory. In Table 1 columns are: target names, positions, number of reference stars, number of frames, proper motions, the interval of time for which we have observations, correction applied to the relative parallax to obtain an absolute parallax, absolute parallax estimates and literature values when available. Note the proper motions are essentially relative, not absolute, hence any use of these proper motions should be made with care.

To exhibit the variation in coverage in Fig. 2 we plot the solutions of GJ 1167 A and LHS 1104 with 14 and 136 observations respectively. The reason for this large difference in observational history is a combination of longer temporal coverage, higher priority (e.g. LHS 1104 as a visual binary has a higher priority), and sporadic access to the telescope.

Measured parallax values of targets in common with Smart et al. (2007) (GJ 1167 A, LHS 2472, LHS 369, LHS 3872 and
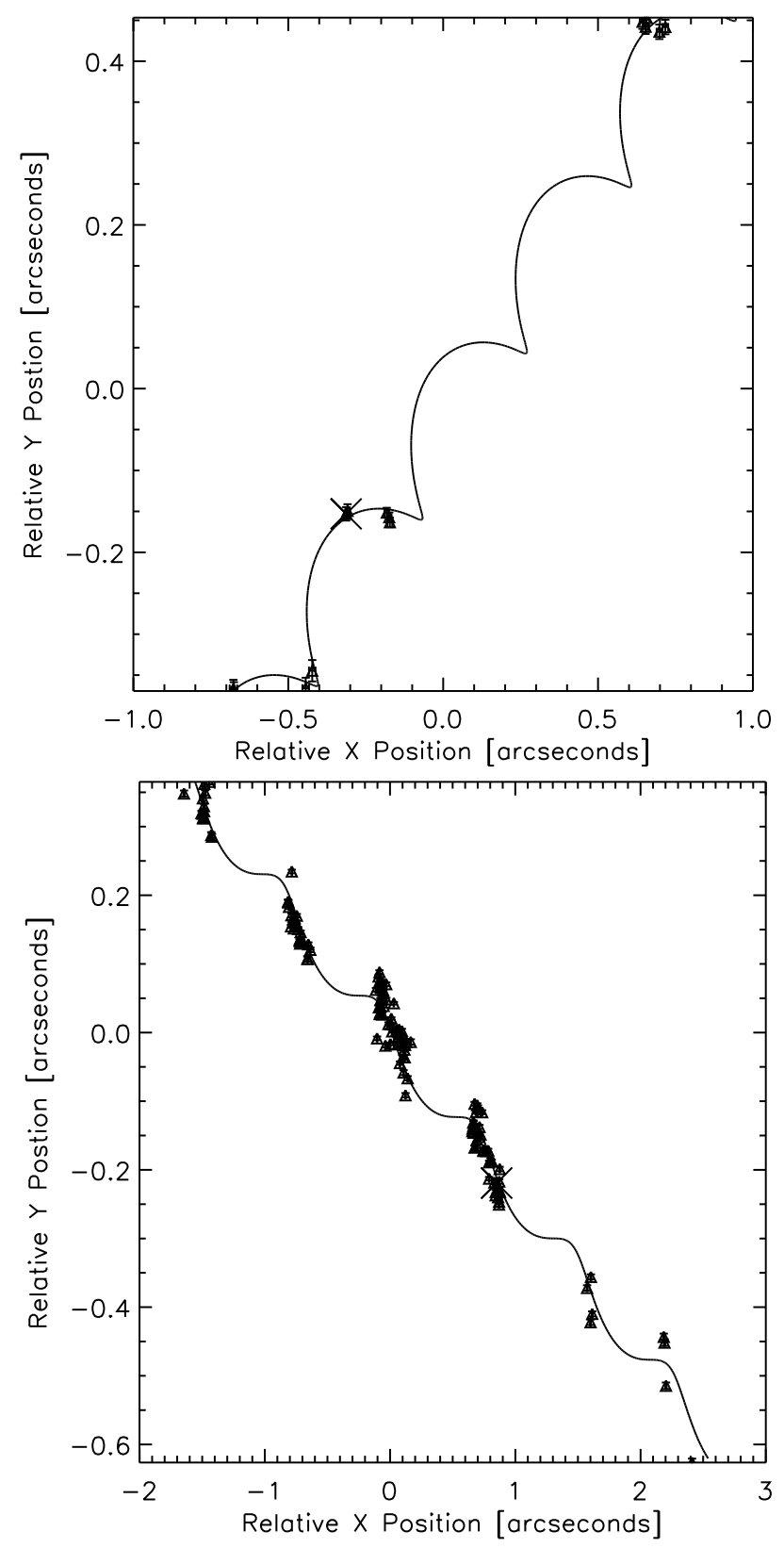

Fig. 2. Observations for two targets showing the range of coverage, 14 observations for GJ 1167 A and 136 for LHS 1104.

LHS 1050) agree within the errors. All other objects agree with literature values to within $2 \sigma$ apart from LHS 1475, LHS 3494, LHS 528 and LHS 535 which are all addressed in the discussion.

\section{Model comparison}

\subsection{Photometry}

We attempted to compile a set of uniform magnitudes in the $V, R_{\mathrm{c}}, I_{\mathrm{c}}, J, H, K_{\mathrm{s}}$ (hereafter the Cousins bands will be simply denoted $R$ and $I$ ) for the comparison to theoretical models. The photometric data are from various sources, where available we used photoelectric values from the literature. As part of the TOPP program, many fields were observed in the $V$ and $I$ bands for the DCR correction and transformed to a standard system using procedures described in Bucciarelli et al. (2001). When an object had no photometry in the literature we took the $V, I$ from 
Table 1. Parallaxes and proper motions for TOPP red dwarfs.

\begin{tabular}{|c|c|c|c|c|c|c|c|c|c|c|}
\hline ID, LHS & GJ & $\begin{array}{c}\text { RA } \\
\text { (h:m:s ) }\end{array}$ & $\begin{array}{c}\text { Dec } \\
\left({ }^{\circ}:^{\prime}:^{\prime \prime}\right) \\
\end{array}$ & $N_{*}, N_{f}$ & $\begin{array}{r}\mu_{\alpha} \\
(\mathrm{mas} / \mathrm{yr}) \\
\end{array}$ & $\begin{array}{r}\mu_{\delta} \\
\text { (mas/yr) } \\
\end{array}$ & $\begin{array}{r}\Delta T \\
(\mathrm{yrs})\end{array}$ & $\begin{array}{r}\text { COR } \\
\text { (mas) }\end{array}$ & $\begin{array}{r}\pi \\
(\mathrm{mas}) \\
\end{array}$ & $\begin{array}{r}\text { Literature } \pi \\
\text { (mas) }\end{array}$ \\
\hline 1,1047 & 1005 & $0: 15: 28.2$ & $-16: 7: 43.5$ & 7,37 & $596.8 \pm 4.7$ & $-629.0 \pm 5.1$ & 3.29 & 2.05 & $179.8 \pm 11.6$ & $191.9 \pm 17.2^{1}$ \\
\hline 2,1050 & 12 & $0: 15: 52.3$ & $+13: 32: 41.0$ & 8,83 & $606.7 \pm 1.4$ & $336.2 \pm 1.5$ & 5.20 & 1.86 & $87.4 \pm 3.4$ & $84 \pm 11^{2}$ \\
\hline 3,1104 &.. & $0: 35: 47.9$ & $+52: 41: 41.7$ & 33,136 & $775.9 \pm 1.0$ & $-174.3 \pm 1.5$ & 5.20 & 1.05 & $40.5 \pm 2.2$ & \\
\hline 4,1475 & $119 \mathrm{~A}$ & $2: 56: 29.6$ & $+55: 26: 1.2$ & 33,108 & $723.9 \pm 2.6$ & $-444.3 \pm 2.8$ & 5.20 & 0.44 & $59.9 \pm 5.0$ & $30.94 \pm 9.86^{1}$ \\
\hline 5,228 & 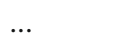 & $7: 16: 21.9$ & $+23: 42: 32.4$ & 24,171 & $936.1 \pm 0.4$ & $-571.1 \pm 0.4$ & 6.30 & 1.30 & $23.6 \pm 1.1$ & $17.8 \pm 3.0^{3}$ \\
\hline 6,1923 & 3444 & $7: 32: 10.7$ & $+57: 55: 33.7$ & 16,145 & $2.7 \pm 0.6$ & $-914.2 \pm 0.7$ & 6.02 & 2.27 & $44.0 \pm 1.7$ & \\
\hline 7,243 & $\ldots$ & 8: $3: 7.1$ & $+34: 55: 59.6$ & 11,113 & $-512.7 \pm 0.7$ & $-1494.0 \pm 0.8$ & 3.29 & 1.61 & $41.0 \pm 1.2$ & \\
\hline 8,1976 & $\ldots$ & $8: 3: 7.1$ & $+52: 50: 38.0$ & 12,33 & $362.3 \pm 2.3$ & $-668.2 \pm 2.1$ & 4.11 & 1.11 & $40.3 \pm 5.1$ & $32.07 \pm 4.26^{1}$ \\
\hline 9,2065 & 3517 & $8: 53: 46.0$ & $-3: 29: 36.0$ & 11,23 & $-507.1 \pm 1.5$ & $-189.9 \pm 2.8$ & 4.93 & 1.66 & $109.9 \pm 4.6$ & $113.3 \pm 2.0^{4}$ \\
\hline 10,306 & 3668 & $11: 31: 26.7$ & $-14: 58: 17.0$ & 8,14 & $401.3 \pm 5.6$ & $-1378.2 \pm 5.2$ & 6.02 & 1.78 & $85.0 \pm 19.2$ & $89.24 \pm 1.69^{7}$ \\
\hline 11,2472 & 452.1 & 54: 8.1 & $+9: 48: 11.9$ & 7,36 & $90.0 \pm 1.5$ & $-791.0 \pm 1.5$ & 6.02 & 1.91 & $3 \pm 3.7$ & $92 \pm 12^{5}$ \\
\hline 12,326 & $\ldots$ & $24: 23.2$ & $-4: 43: 47.2$ & 13,54 & $1142.3 \pm 1.7$ & $-635.8 \pm 1.6$ & 4.11 & 2.10 & \pm 4.3 & ... \\
\hline 13,334 & $\ldots$ & $12: 34: 14.5$ & $+20: 36: 33.4$ & 8,34 & $333.7 \pm 1.3$ & $-1294.8 \pm 1.9$ & 5.20 & 2.42 & $1 \pm 3.9$ & $\ldots$ \\
\hline 14,2632 & & $12: 46: 50.8$ & $+31: 47: 57.0$ & 12,127 & $-796.6 \pm 0.8$ & $40.9 \pm 0.6$ & 6.30 & 2.05 & $53.3 \pm 2.3$ & \\
\hline $15, \ldots$ & $1167 \mathrm{~A}$ & 13: 9:32.1 & $+28: 56: 45.0$ & 7,14 & $-337.6 \pm 1.8$ & $-207.9 \pm 2.9$ & 3.83 & 1.88 & $92.5 \pm 5.0$ & $84 \pm 12^{6}$ \\
\hline 16,2686 & $\ldots$ & 13: 9:59.7 & $+47: 44: 40.5$ & 5,42 & $-634.2 \pm 1.2$ & $-606.7 \pm 1.3$ & 6.30 & 1.80 & $96.8 \pm 4.7$ & $\ldots$ \\
\hline 17,2719 & $\ldots$ & $13: 20: 32.2$ & $-3: 55: 23.0$ & 5,64 & $-850.4 \pm 0.9$ & $287.5 \pm 1.3$ & 6.57 & 2.00 & $37.1 \pm 3.4$ & $\cdots$ \\
\hline 18,360 & $\ldots$ & $13: 46: 53.6$ & $+5: 43: 5.2$ & 11,33 & $-767.9 \pm 1.0$ & $-848.0 \pm 1.3$ & 4.38 & 2.05 & $17.8 \pm 3.0$ & $10.33 \pm 2.85^{4}$ \\
\hline 19,369 & 545 & $14: 20: 7.5$ & $-9: 35: 49.4$ & 8,28 & $-606.7 \pm 1.3$ & $-829.2 \pm 2.1$ & 5.20 & 1.85 & $69.0 \pm 4.0$ & $71.52 \pm 1.38^{7}$ \\
\hline 20,2924 & 3849 & $14: 28: 36.2$ & $+33: 10: 47.9$ & 10,29 & $-338.0 \pm 2.9$ & $-714.8 \pm 2.5$ & 4.38 & 1.15 & $84.5 \pm 5.5$ & $90.0 \pm 1.3^{8}$ \\
\hline 21,3343 & 4040 & $17: 57: 50.9$ & $+46: 34: 53.3$ & 9,155 & $-10.7 \pm 0.6$ & $592.0 \pm 1.0$ & 6.02 & 1.99 & $68.4 \pm 2.3$ & $73.75 \pm 1.84^{1}$ \\
\hline 22,3445 & $9652 \mathrm{~A}$ & 19:14:37.5 & $+19: 18: 31.2$ & 23,153 & $-630.7 \pm 0.8$ & $441.2 \pm 1.3$ & 6.30 & 0.41 & $55.7 \pm 2.3$ & $52.45 \pm 2.75^{1}$ \\
\hline 23,3482 & $767 \mathrm{~A}$ & $19: 46: 15.1$ & $+32: 1: 0.8$ & 74,93 & $463.9 \pm 1.1$ & $-393.8 \pm 1.7$ & 6.02 & 0.28 & $68.6 \pm 3.4$ & $74.90 \pm 2.93^{1}$ \\
\hline 24,3494 & $1245 \mathrm{~A}$ & $19: 53: 48.9$ & $+44: 24: 56.5$ & 15,113 & $410.9 \pm 3.2$ & $-469.9 \pm 1.5$ & 4.11 & 0.52 & $227.9 \pm 3.9$ & $219.2 \pm 1.4^{9}$ \\
\hline 25,528 & 1271 & $22: 42: 43.4$ & $+17: 40: 12.1$ & 9,104 & $1105.0 \pm 0.9$ & $528.4 \pm 1.1$ & 5.20 & 1.70 & $57.6 \pm 2.4$ & $47.13 \pm 2.96^{1}$ \\
\hline 26,3872 & 4302 & $22: 54: 38.4$ & $-5: 27: 59.7$ & 6,32 & $609.9 \pm 1.5$ & $354.3 \pm 2.0$ & 5.20 & 2.19 & $40.6 \pm 4.6$ & \\
\hline 27,535 & 4312 & $23: 7: 42.2$ & $+68: 41: 51.5$ & 40,70 & $1139.0 \pm 1.3$ & $60.3 \pm 1.4$ & 5.48 & 0.78 & $76.7 \pm 2.8$ & $63.5 \pm 4.2^{10}$ \\
\hline
\end{tabular}

References. ${ }^{(1)}$ Perryman et al. (1997); (2) van Maanen (1944); (3) Harrington et al. (1985); (4) Costa et al. (2005); ${ }^{(5)}$ Stearns (1959); ${ }^{(6)}$ Heintz (1994); (7) Jao et al. (2005); ${ }^{(8)}$ Monet et al. (1992); ${ }^{(9)}$ Harrington et al. (1993); ${ }^{(10)}$ Dahn et al. (1982) .

Notes. $N_{*}=$ number of reference stars; $N_{f}=$ number of frames; $\Delta T=$ epoch range; $\mathrm{COR}=$ correction to absolute parallax.

the TOPP observations. Overall, we obtained photoelectric photometry on the standard $V, R, I$ systems for around $90 \%$ of our objects. The errors for the different sources varied from 0.02 to $0.08 \mathrm{mag}$.

For the remaining missing magnitudes we used photographic magnitudes from the GSC2.3 catalogue. The GSC2.3 system is described in Lasker et al. (2008) and within the GSC-II consortium there are a set of standard transformations from the Johnson-Cousins system to natural GSC-II plate bandpass. However, these transformations apply to stars and the $\mathrm{M}$ dwarfs under study here have extreme colors, so we constructed a transformation calibrated on an identical sample. To do this we took a sample of $\mathrm{M}$ dwarfs from the RECONS program and the Hipparcos catalogue to determine a linear transformation between the GSC2.3 magnitudes, $V_{\mathrm{pg}}, R_{\mathrm{F}}$ and $I_{\mathrm{N}}$ and the JohnsonCousins magnitudes $V, R, I$ viz:

$$
\begin{aligned}
V & =V_{\mathrm{pg}}+(0.566 \pm 0.226)-\left((0.384 \pm 0.215) *\left(V_{\mathrm{pg}}-R_{\mathrm{F}}\right)\right) \\
R & =R_{\mathrm{F}}+(0.515 \pm 0.022)-\left((0.397 \pm 0.013) *\left(R_{\mathrm{F}}-I_{\mathrm{N}}\right)\right) \\
I & =I_{\mathrm{N}}+(-0.006 \pm 0.033)-\left((0.046 \pm 0.011) *\left(R_{\mathrm{F}}-I_{\mathrm{N}}\right)\right)
\end{aligned}
$$

The $R_{\mathrm{F}}$ and $I_{\mathrm{N}}$ filters transformations are well determined while the $V_{\text {pg }}$ filter is not. In Table 2 we indicate those magnitudes obtained from this transformation with the index ${ }^{5}$.

All the infrared magnitudes come from the 2MASS catalogue. As these are in general more precise than the optical measures and on a more consistent system, we limit the use of the optical magnitudes to the color axis and when possible use $R-K_{\mathrm{s}}$ to give us a large baseline.

In Fig. 3 plots of $R-I$ against $H-K_{\mathrm{s}}$ have been made for TOPP stars to compare with Leggett (1992) mean observed colours for red dwarfs. The $H-K$ values of Leggett (1992) are in CIT system and they have been transformed to 2MASS values using the following transformation from Carpenter (2001). $\left(K_{\mathrm{S}}\right)_{2 \mathrm{MASS}}=K_{\mathrm{CIT}}+(0.000 \pm 0.005)(J-K)_{\mathrm{CIT}}-(0.024 \pm 0.003)$ $\left(H-K_{\mathrm{s}}\right)_{2 \mathrm{MASS}}=(1.026 \pm 0.020)(H-K)_{\mathrm{CIT}}+(0.028 \pm 0.005) \mathrm{As}$ a comparison sample we selected 797 stars from the Hipparcos, RECONS and USNO catalogues with the following criteria: $\pi / \sigma_{\pi}>5, \pi>20$ mas, and $V_{J}>6$. Figure 4 shows the Colour - Magnitude diagram for TOPP stars and this reference sample. The spread seen is partially due to errors but also intrinsic to the sample.

\subsection{Theoretical models}

We compared a number of Color-Magnitude relations to theoretical model simulations to find parameters for our targets. All simulations were generated using the Phoenix web simulator (http://phoenix.ens-lyon. fr/simulator) with the NextGen model (Hauschildt et al. 1999) for metallicities $[\mathrm{M} / \mathrm{H}]=0.0,-0.5,-1.0$ and -1.5 . We generated directly Cousins $R I$ and $2 \mathrm{MASS} J H K_{\mathrm{s}}$ magnitudes to avoid possible transform problems. As examples we plot TOPP results with low and high metallicity simulations with equal mass, Fig. 5, and 
A\&A 514, A84 (2010)

Table 2. Photometric data and absolute magnitudes based on the parallaxes in Table 1.

\begin{tabular}{|c|c|c|c|c|c|c|c|c|c|}
\hline ID & LHS & $V$ & $R$ & $I$ & $J$ & $H$ & $K_{\mathrm{s}}$ & $M(J)$ & $M(K)$ \\
\hline 1 & 1047 & $11.48^{1}$ & $10.27^{1}$ & $8.71^{1}$ & 7.22 & 6.71 & 6.39 & $8.49(-0.14,+0.14)$ & $7.66(-0.14,+0.14)$ \\
\hline 2 & 1050 & $12.61^{2}$ & $11.46^{2}$ & $10.04^{2}$ & 8.62 & 8.07 & 7.81 & $8.33(-0.08,+0.09)$ & $7.52(-0.08,+0.09)$ \\
\hline 3 & 1104 & $12.54^{3}$ & $11.35^{3}$ & $10.34^{3}$ & 8.93 & 8.35 & 8.10 & $6.97(-0.12,+0.12)$ & $6.13(-0.12,+0.12)$ \\
\hline 4 & 1475 & $10.48^{3}$ & $9.50^{3}$ & $8.71^{3}$ & 7.43 & 6.80 & 6.59 & $6.31(-0.17,+0.19)$ & $5.48(-0.17,+0.19)$ \\
\hline 5 & 228 & $15.53^{4}$ & $14.42^{5}$ & $13.25^{4}$ & 12.02 & 11.52 & 11.3 & $8.89(-0.10,+0.11)$ & $8.17(-0.10,+0.11)$ \\
\hline 6 & 1923 & $18.09^{4}$ & $15.91^{5}$ & $13.94^{4}$ & 11.92 & 11.38 & 11.09 & $10.14(-0.08,+0.08)$ & $9.31(-0.08,+0.08)$ \\
\hline 7 & 243 & $16.09^{6}$ & $14.80^{6}$ & $13.12^{6}$ & 11.51 & 11.04 & 10.74 & $9.58(-0.06,+0.07)$ & $8.81(-0.06,+0.07)$ \\
\hline 8 & 1976 & $11.38^{3}$ & $10.31^{3}$ & $9.41^{3}$ & 8.06 & 7.48 & 7.24 & $6.08(-0.26,+0.29)$ & $5.26(-0.26,+0.29)$ \\
\hline 9 & 2065 & $18.96^{7}$ & $16.78^{7}$ & $14.49^{7}$ & 11.21 & 10.47 & 9.94 & $11.42(-0.09,+0.09)$ & $10.1(-0.09,+0.09)$ \\
\hline 10 & 306 & $14.19^{8}$ & $12.80^{8}$ & $11.05^{8}$ & 9.36 & 8.76 & 8.50 & $9.00(-0.44,+0.56)$ & $8.14(-0.44,+0.56)$ \\
\hline 11 & 2472 & $12.81^{2}$ & $11.61^{2}$ & $10.12^{2}$ & 8.70 & 8.19 & 7.87 & $8.43(-0.09,+0.09)$ & $7.6(-0.09,+0.09)$ \\
\hline 12 & 326 & $14.93^{9}$ & $13.99^{9}$ & $13.05^{9}$ & 11.93 & 11.43 & 11.23 & $7.06(-0.74,+1.14)$ & $6.36(-0.74,+1.14)$ \\
\hline 13 & 334 & $18.02^{10}$ & $16.70^{10}$ & $15.13^{10}$ & 13.75 & 13.25 & 13.04 & $10.47(-0.35,+0.42)$ & $9.77(-0.35,+0.42)$ \\
\hline 14 & 2632 & $19.15^{4}$ & $16.80^{5}$ & $14.76^{4}$ & 12.23 & 11.58 & 11.21 & $10.86(-0.09,+0.10)$ & $9.84(-0.09,+0.10)$ \\
\hline 15 & $\ldots$ & $14.52^{3}$ & $13.35^{3}$ & $11.88^{3}$ & 9.48 & 8.91 & 8.61 & $9.31(-0.11,+0.12)$ & $8.44(-0.11,+0.12)$ \\
\hline 16 & 2686 & $14.16^{11}$ & $12.88^{11}$ & $11.18^{11}$ & 9.58 & 9.00 & 8.69 & $9.51(-0.10,+0.11)$ & $8.62(-0.10,+0.11)$ \\
\hline 17 & 2719 & & $14.84^{5}$ & $13.09^{5}$ & 11.28 & 10.77 & 10.48 & $9.12(-0.19,+0.21)$ & $8.33(-0.19,+0.21)$ \\
\hline 18 & 360 & $15.22^{9}$ & $14.29^{9}$ & $13.41^{9}$ & 12.39 & 11.85 & 11.62 & $8.64(-0.34,+0.40)$ & $7.87(-0.34,+0.40)$ \\
\hline 19 & 369 & $12.96^{2}$ & $11.75^{2}$ & $10.25^{2}$ & 8.74 & 8.19 & 7.98 & $7.93(-0.12,+0.13)$ & $7.17(-0.12,+0.13)$ \\
\hline 20 & 2924 & $19.58^{12}$ & $17.28^{5}$ & $15.21^{12}$ & 11.99 & 11.23 & 10.74 & $11.62(-0.14,+0.15)$ & $10.3(-0.14,+0.15)$ \\
\hline 21 & 3343 & $11.68^{3}$ & $10.42^{3}$ & $9.32^{3}$ & 7.85 & 7.25 & 7.00 & $7.02(-0.07,+0.07)$ & $6.17(-0.07,+0.07)$ \\
\hline 22 & 3445 & $11.59^{3}$ & $10.31^{3}$ & $9.16^{3}$ & 7.58 & 7.03 & 6.81 & $6.31(-0.09,+0.09)$ & $5.54(-0.09,+0.09)$ \\
\hline 23 & 3482 & $9.79^{3}$ & $8.71^{3}$ & $7.82^{3}$ & 6.88 & 6.22 & 6.04 & $6.06(-0.11,+0.11)$ & $5.22(-0.11,+0.11)$ \\
\hline 24 & 3494 & $13.41^{3}$ & $11.41^{3}$ & $9.76^{3}$ & 7.79 & 7.19 & 6.85 & $9.58(-0.04,+0.04)$ & $8.64(-0.04,+0.04)$ \\
\hline 25 & 528 & $11.76^{3}$ & $10.56^{3}$ & $9.52^{3}$ & 8.06 & 7.38 & 7.18 & $6.86(-0.09,+0.09)$ & $5.98(-0.09,+0.09)$ \\
\hline 26 & 3872 & $13.87^{3}$ & $12.43^{3}$ & $11.20^{3}$ & 9.65 & 9.09 & 8.81 & $7.69(-0.23,+0.26)$ & $6.85(-0.23,+0.26)$ \\
\hline 27 & 535 & $12.45^{3}$ & $11.20^{3}$ & $10.10^{3}$ & 8.62 & 8.10 & 7.92 & $8.05(-0.08,+0.08)$ & $7.34(-0.08,+0.08)$ \\
\hline
\end{tabular}

References. ${ }^{(1)}$ Kilkenny et al. (2007); (2) Bessell (1990); (3) Weis (1996); (4) TOPP see text ; ${ }^{(5)}$ GSC2.3 transformation see text ; ${ }^{(6)}$ Dawson \& Forbes (1989); (7) Costa et al. (2005); ${ }^{(8)}$ Jao et al. (2005); ${ }^{(9)}$ Costa et al. (2006); ${ }^{(10)}$ Jao et al. (2008); ${ }^{(11)}$ Weistrop (1979); ${ }^{(12)}$ Dahn et al. (2002) . Notes. All infrared magnitudes come from the 2MASS catalogue. Absolute magnitude uncertainties include both parallax and apparent magnitude errors.

age, Fig. 6, contours. It is clear that different values of metallicity are needed to describe our stars. We did not feel the precision of the models or of the observations warranted a finer grid in metallicity, which sometimes lead to conflicting results, such as subdwarfs with a young age. Our procedure is to try to find the closest to solar metallicity main sequence solution and then seek other solutions if no solar metallicity solution can be found. From the various Colour - Magnitude diagram and theoretical model comparisons we estimate masses and ages as shown in Table 3. In this table we also include tangential velocities derived from the TOPP results and estimated spectral types taken from the references listed. Finally, we check our observational sequence for any possible variability and include this in the discussion when relevant.

Metallicity assumptions influence the inferred masses and ages. For example the M3.5 LHS 1050 (object number 2 in Figs. 5 and 6$)$ is assigned $[\mathrm{m} / \mathrm{H}]=0$. If it is in fact more metalpoor with $[\mathrm{m} / \mathrm{H}]=-0.5$ then the assigned age would be younger (0.02-0.04 Gyr instead of $0.125-10 \mathrm{Gyr}$ ) and the mass would be lower $\left(0.09-0.12 M_{\odot}\right.$ instead of $\left.0.21-0.23 M_{\odot}\right)$. Similarly, if the metallicity is higher, an older age and higher mass would be determined. For example if LHS 228 (object number 5) has a metallicity of -0.5 instead of -1 , then the mass would be $0.13-0.175 M_{\odot}$ instead of $0.10-0.14 M_{\odot}$ and the age would be 10 Gyr instead of $0.05-0.20$ Gyr. The effect is not a strong function of spectral type; for the M6 LHS 1923 (object number 6), if the metallicity is $[\mathrm{m} / \mathrm{H}]=-0.5$ instead of $[\mathrm{m} / \mathrm{H}]=0$, then again the assigned age would be very young (0.1-0.2 Gyr instead of $0.4-10 \mathrm{Gyr})$ and the mass would be lower $\left(0.075 M_{\odot}\right.$ instead of $\left.0.1 M_{\odot}\right)$. Hence if the metallicity is unknown to $0.5 \mathrm{dex}$, age is effectively undetermined and mass is uncertain to about a factor of two.

\section{Discussion}

We note that nearly half the sample in Table 3 is predicted to be younger than 0.5 Gyr. There may be weak biases in our sample selection. The high proper motion selection could produce a bias to older objects. The choice of bright targets suitable for a $1 \mathrm{~m}$ telescope may produce a bias to younger objects. However we do not believe this explains the apparently young ages in Table 3. A systematic underestimate of the metallicity (i.e. assigning a metallicity that is too low) could produce ages that are too young (see Sect. 4.2). This may suggest that a significant number of the nearby $\mathrm{M}$ dwarfs are metal-rich (e.g. Fig. 2 of Johnson \& Apps 2009), or it may reflect a systematic error in the modelled $R$ and $K$ magnitudes due to the known problems with the calculated opacities for cool atmospheres (Lyubchik et al. 2007).

The range of masses and ages in Table 3 are estimated using only the uncertainties in the parallaxes and photometry, and do not include the systematic uncertainties due to model selection, in particular metallicity. As we show in Sect. 4.2 including this uncertainty implies that the age is not well determined and the masses could differ by a factor of two from what is listed in 


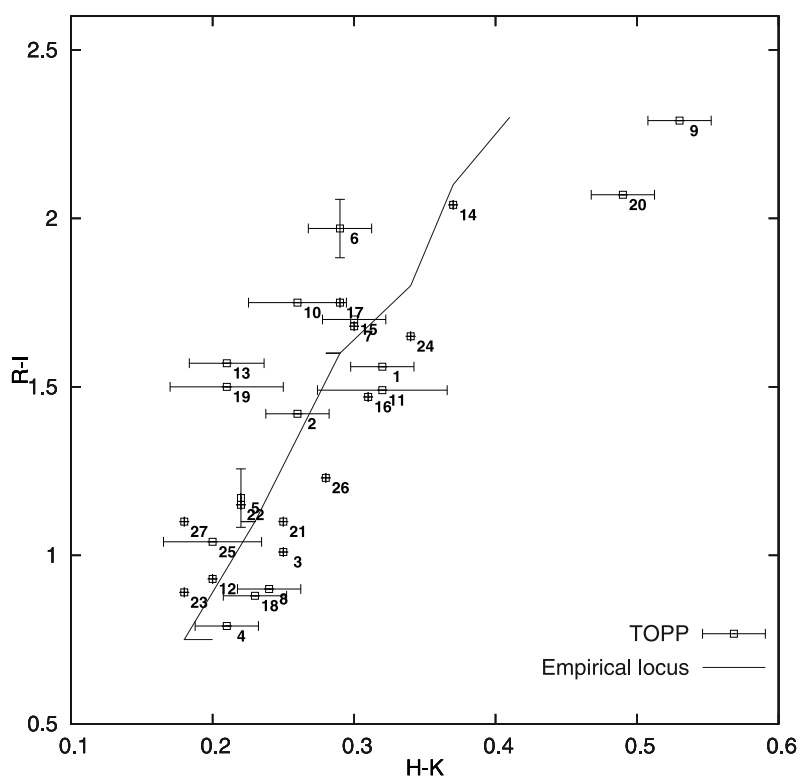

Fig. 3. $R-I$ versus $H-K_{\mathrm{s}}$ diagram for the TOPP stars with identifiers as listed in Table 1. The continuous line is the mean observed colours from Leggett (1992).

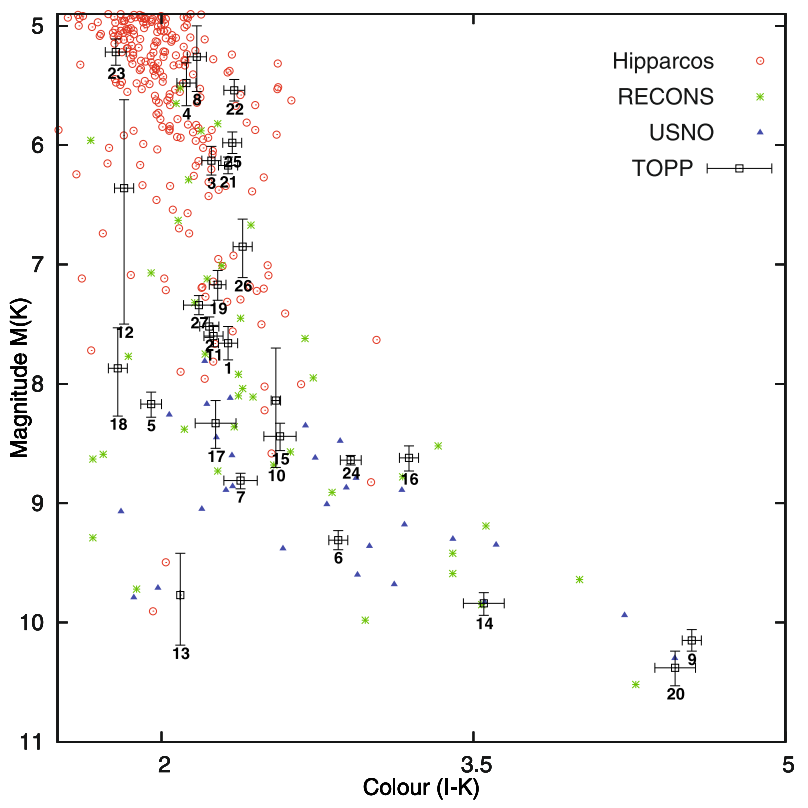

Fig. 4. Colour-Magnitude diagram $\left(M(K)\right.$ vs. $\left.I-K_{\mathrm{s}}\right)$ of the TOPP stars identified as listed in Table 1, with Hipparcos, RECONS and USNO objects plotted for reference.

the table. We now consider groups of objects that appear to be similar.

LHS 1050, LHS 1104, LHS 1923, LHS 306, LHS 2472, GJ 1167 A, LHS 2686, LHS 2719, LHS 369, LHS 3343, LHS 3482, LHS 3872, LHS 528 are consistent with being low-mass main-sequence stars with solar metallicities. The LHS 528 parallax is over $2 \sigma$ from the Hipparcos value but the observational sequence is solid and there is no evidence for binary motion, so we cannot explain this inconsistency. However, we note that, based on the $V$ magnitude of 11.76 , this object is at the faint limit of the Hipparcos catalogue.
LHS 1047, LHS 1976 are consistent with being low-mass, single, main-sequence stars with solar metallicities, however, the errors on the parallaxes are larger than average. LHS 1047 was discovered by Ianna et al. (1988) to be a binary system, speckle measurements by Docobo et al. (2006) put the secondary 218 mas away from the primary and 1.8 mag fainter. LHS 1976 is seen in the Hipparcos data as a binary system separated by 270 mas and speckle interferometric observations (Balega et al. 2004) also suggest the presence of a third component 40 mas from the secondary and 0.27 mag fainter. If we assume the published magnitude differences the derived primary age range will be increased from (0.08-0.17) to (0.1-0.2) Gyr for LHS 1047 and (0.055-0.1) to (0.2-10) Gyr for LHS 1976; the mass range will not vary for LHS 1047 while for LHS 1976 it will decrease from $(0.53-0.61)$ to $(0.48-0.56) M_{\odot}$. This does not take into account the effect of the unmodelled multiplicity on our parallax determination which is also probably the cause of the large parallax errors.

LHS 2065, LHS 2632, LHS 2924 are objects close to the end of the main sequence with very low masses (0.06-0.095), indicating that they are around the transition between low-mass stars and brown dwarfs. All masses, ages and errors are based on assuming solar metallicity. LHS 2065 and LHS 2924 are catalogued as flare stars but the only evidence of variability over the 4 years of TOPP observations is a gradual dimming for LHS 2065 by $0.06+/-0.02$ mag. LHS 2065 and LHS 2924 are also the reddest objects in our study and in fact they are outliers in Fig. 3; this can be most readily explained by the presence of dust in their atmospheres (e.g. Jones \& Tsuji 1997). The lack of lithium in LHS 2065 (Martín et al. 1999) indicates that the mass of this object is greater than $0.06 M_{\odot}$.

LHS 1475 is a main sequence star in a visual binary system with LHS 1476. We note that the TOPP parallax is twice the Hipparcos value; we believe this is because during the Hipparcos mission this object moved in front of another bright star and was effected by veiling glare (Froeschle et al. 1985). This is also reflected in the large errors of the Hipparcos values. The Yale Parallax Catalog lists five published individual parallaxes: $76.9 \pm$ 17.8 and $60 \pm 15.0$ from the $\mathrm{M}^{c}$ Cormick Observatory, $43.2 \pm 5.0$ and $36.9 \pm 5.7$ from the Sproul Observatory and $46.3 \pm 21.6$ from the Pulkovo Observatory. We note that the TOPP value is more consistent with these values than the Hipparcos value but we postpone any further conclusions until a binary solution is made including LHS 1476.

LHS 3494 was cataloged as a double system by McCarthy et al. (1988) with a secondary that is $1.1 \mathrm{mag}$ fainter. If we assume this magnitude differences the derived primary age range will be increased from $(0.12-0.2)$ to $(0.2-10$.) Gyr and the mass range decreased from $(0.09-0.11)$ to $(0.11-0.12) M_{\odot}$. It is also a known flare star and indeed the photometric standard deviation for the TOPP sequence was approximately twice that of the other objects in this study. The TOPP parallax is inconsistent at the $2 \sigma$ level with that found by Harrington et al. (1993), which may be due to the unmodelled binary nature.

LHS 3445 The position of this object suggests either a young star or a close binary system. Since this is part of a visual binary system with LHS 3446 and we have used a simple single 
Table 3. Derived parameters from model comparisons.

\begin{tabular}{|c|c|c|c|c|c|}
\hline ID, LHS & $\begin{array}{l}\text { MK } \\
\text { Type }\end{array}$ & $\mathrm{M} / \mathrm{H}$ & $\begin{array}{l}\text { Mass } \\
\left(M_{\odot}\right)\end{array}$ & $\begin{array}{l}\text { Age } \\
\text { (Gyr) }\end{array}$ & $\begin{array}{l}V_{\mathrm{tan}} \\
\left(\mathrm{km} \mathrm{s}^{-1}\right)\end{array}$ \\
\hline $1,1047^{*}$ & $\mathrm{M} 4^{1}$ & 0 & $0.16-0.18$ & $0.08-0.17$ & $22 \pm 1$ \\
\hline 2,1050 & M3. $5^{1}$ & 0 & $0.21-0.23$ & $0.125-10$ & $37 \pm 1$ \\
\hline 3,1104 & & 0 & $0.42-0.46$ & $0.12-10$ & $59 \pm 3$ \\
\hline 4,1475 & $\mathrm{M} 1^{1}$ & 0 & $0.55-0.58$ & $0.12-0.30$ & $48 \pm 4$ \\
\hline 5,228 & & -1 & $0.10-0.14$ & $0.05-0.20$ & $206 \pm 10$ \\
\hline 6,1923 & $\mathrm{M} 6^{2}$ & 0 & $0.10-0.11$ & $0.4-10$ & $98 \pm 4$ \\
\hline 7,243 & & -.5 & $0.08-0.11$ & $0.11-0.25$ & $179 \pm 5$ \\
\hline $8,1976^{*}$ & & 0 & $0.53-0.61$ & $0.055-10$ & $83 \pm 10$ \\
\hline 9,2065 & $M 9 e^{3}$ & 0 & $0.06-0.07$ & $0.25-0.45$ & $23 \pm 1$ \\
\hline 10,306 & M5. $5^{4}$ & 0 & $0.10-0.13$ & $0.04-10$ & $80 \pm 18$ \\
\hline 11,2472 & M3. $5^{1}$ & 0 & $0.18-0.21$ & $0.12-10$ & $43 \pm 2$ \\
\hline 12,326 & & -.5 & $0.34-0.44$ & $0.035-10$ & $582 \pm 238$ \\
\hline 13,334 & & -1 & $0.09-0.10$ & $0.3-10$ & $286 \pm 51$ \\
\hline 14,2632 & M7. $5^{5}$ & 0 & $0.08-0.10$ & $0.6-10$ & $60 \pm 3$ \\
\hline $15, \ldots$ & $\mathrm{M} 4^{1}$ & 0 & $0.11-0.14$ & $0.12-10$ & $19 \pm 1$ \\
\hline 16,2686 & & 0 & $0.08-0.10$ & $0.09-0.18$ & $36 \pm 2$ \\
\hline 17,2719 & & 0 & $0.14-0.16$ & $0.2-10$ & $114 \pm 11$ \\
\hline 18,360 & & -1 & $0.20-0.35$ & $0.2-10$ & $304 \pm 51$ \\
\hline 19,369 & $\mathrm{M} 4^{1}$ & 0 & $0.18-0.22$ & $0.060-0.105$ & $70 \pm 4$ \\
\hline 20,2924 & $\mathrm{M}^{3} \mathrm{e}^{3}$ & 0 & $0.07-0.08$ & $0.6-10$ & $43 \pm 3$ \\
\hline 21,3343 & $\mathrm{M} 3^{6}$ & 0 & $0.37-0.42$ & $0.070-0.105$ & $41 \pm 1$ \\
\hline $22,3445^{*}$ & $\mathrm{M} 3^{1}$ & 0 & $0.36-0.45$ & $0.03-0.05$ & $63 \pm 3$ \\
\hline 23,3482 & $\mathrm{M} 1.5^{3}$ & 0 & $0.60-0.62$ & $0.2-10$ & $38 \pm 2$ \\
\hline $24,3494^{*}$ & M5.5 $\mathrm{e}^{3}$ & 0 & $0.09-0.11$ & $0.12-0.20$ & $12 \pm 0$ \\
\hline 25,528 & $\mathrm{M} 3^{1}$ & 0 & $0.39-0.45$ & $0.065-0.1$ & $97 \pm 4$ \\
\hline 26,3872 & $\mathrm{M} 4^{6}$ & 0 & $0.23-0.28$ & $0.06-0.125$ & $82 \pm 9$ \\
\hline 27,535 & M3. $5^{6}$ & -.5 & $0.16-0.20$ & $0.05-0.12$ & $26 \pm 1$ \\
\hline
\end{tabular}

References. ${ }^{(1)}$ Bidelman (1985); ${ }^{(2)}$ Fleming et al. (1993); ${ }^{(3)}$ Leggett (1992); ${ }^{(4)}$ Mathioudakis \& Doyle (1991); ${ }^{(5)}$ Kirkpatrick et al. (1995); (6) Gliese \& Jahreiß (1991).

Notes. The mass and age ranges are based only on the errors in the parallax and photometry and do not include the systematic error of uncertain metallicity. The latter can have a large impact on mass and age as described in Sect. 4.2. ${ }^{(*)}$ These are known or suspect unresolved binary systems while the parameters are based on an assumption of being single stars. Unaccounted multiplicity will tend to increase the predicted age and decrease the predicted mass.

star solution it is not possible to see if the fit residuals indicate an unresolved binary companion. Shkolnik et al. (2009) find an age indicator of 60-300 Myr which is inconsistent with our age (30-50 Myr) assuming the object is single. Taking the radial velocity of $-42.3 \mathrm{~km} \mathrm{~s}^{-1}$ from Gizis et al. (2002) combined with the TOPP results and the velocity of the Sun from Dehnen \& Binney (1998) we find a space motion, in $\mathrm{km} / \mathrm{s}$, of $U=-25$ (radially inwards), $V=-26$ (in the direction of Galactic rotation), and $W=69$ (vertically upwards). This velocity is not consistent with other younger population velocities (e.g. see Holmberg et al. 2009; Zuckerman \& Song 2004). Finally, the photometric distance based on the color and apparent magnitude and following Henry et al. (2004) is 12pc which, being less than the trigonometric distance of $18 \mathrm{pc}$, is consistent with a binary system. Our results therefore imply that this object is more probably a binary rather than a very young star. The effect of unaccounted multiplicity will be to increase the predicted age and decrease the predicted mass.

LHS 243, LHS 326 are consistent with $[\mathrm{M} / \mathrm{H}]=-0.5$ models, hence subdwarfs. The parallax error for LHS 326 is very high
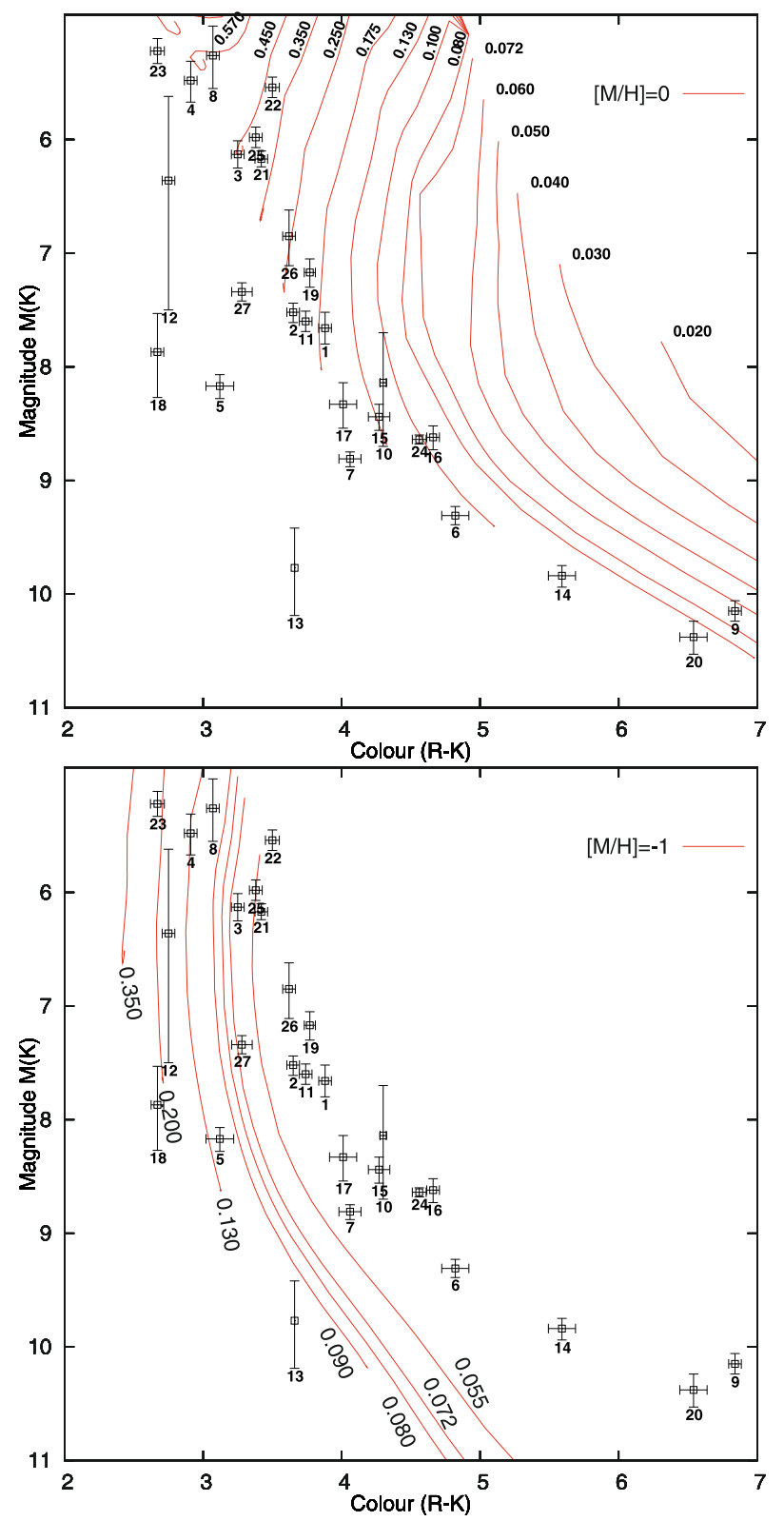

Fig. 5. Colour-Magnitude diagram of the NextGen theoretical models for $[\mathrm{M} / \mathrm{H}]=0$, top panel, and $[\mathrm{M} / \mathrm{H}]=-1.0$, bottom panel. Lines represent different masses (labeled at the end of lines), points are TOPP results with identifiers as shown in Table 1.

but still $2 \sigma$ away from the solar metallicity locus. The position of LHS 243 in Fig. 5 is most consistent with $[\mathrm{M} / \mathrm{H}]=-0.5$; however, if it is closer to $[\mathrm{M} / \mathrm{H}]=0$ then the age range will increase from $0.11-0.25$ to $0.25-10.0 \mathrm{Gyr}$, which is more consistent with our age expectation for a slightly metal poor subdwarf. The high tangential velocities of both objects suggest a non-thin disk membership, supporting this classification.

LHS 535 appears to be most consistent with $[\mathrm{M} / \mathrm{H}]=-0.5$; however, this requires also a very young age and a closer-to-solar metallicity would relax that requirement. We do not believe that the data or models support an investigation using a finer grid, so resolution of this inconsistency will have to wait until better models or spectra are available. We note that the parallax is over $2 \sigma$ from the USNO value but the observational sequence is solid 

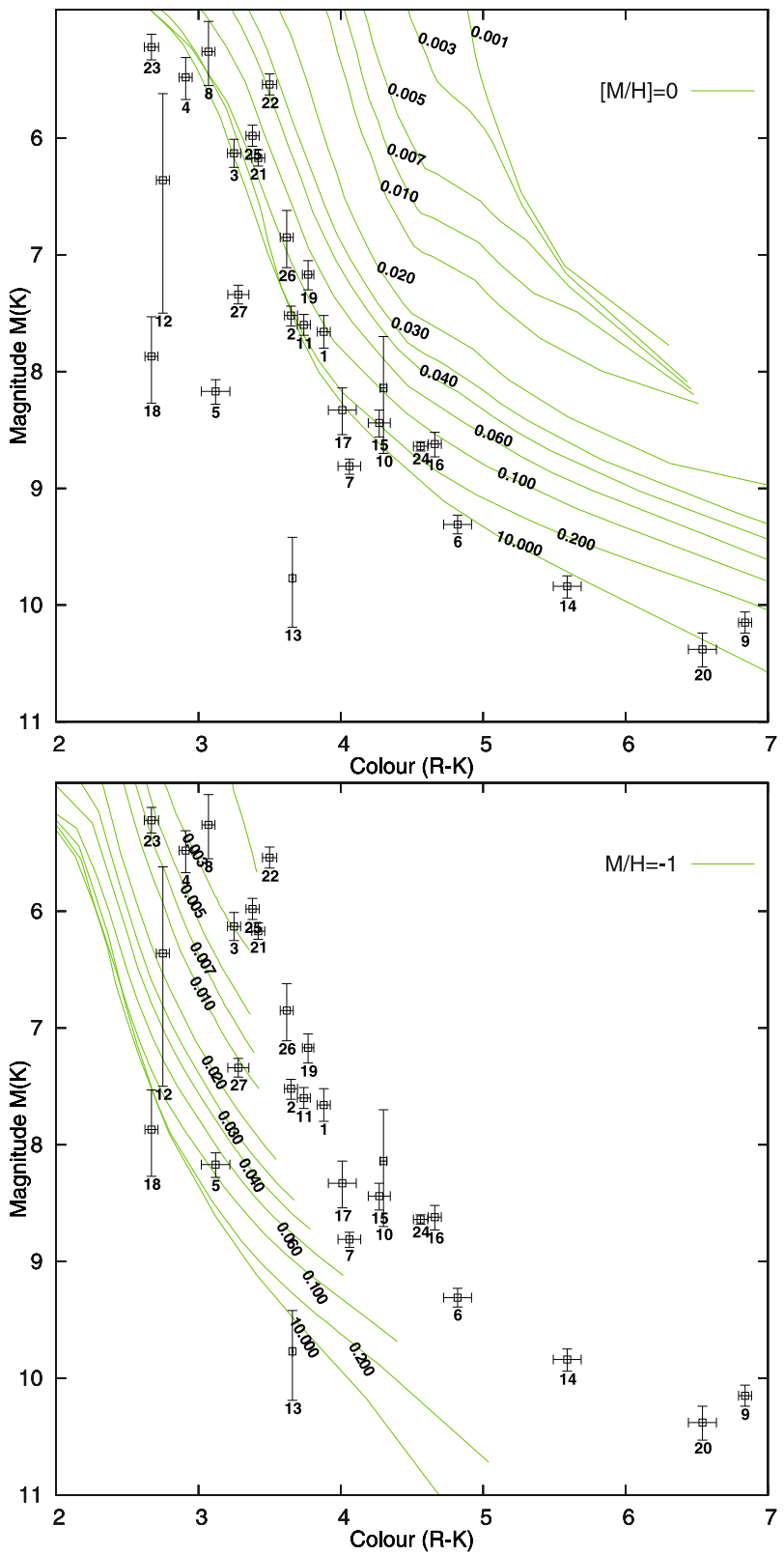

Fig. 6. Colour-Magnitude diagram of the NextGen theoretical models for $[\mathrm{M} / \mathrm{H}]=0$, top panel, and $[\mathrm{M} / \mathrm{H}]=-1.0$, bottom panel. Lines represent different ages in Gyrs (labeled along the lines), points are TOPP results with identifiers as shown in Table 1 .

and there is no evidence for binary motion so we cannot explain the high difference.

LHS 228, LHS 334,LHS 360 The comparison to models suggests that these object are subdwarfs and we base the mass and age values on a $[M / H]=-1$ model. This interpretation is supported by the high tangential velocities. LHS 228 also has a high radial velocity (Dawson \& De Robertis $1989,73 \pm 3 \mathrm{~km} \mathrm{~s}^{-1}$ ), and a spectroscopic classification of subdwarf (Lépine et al. 2007). The metallicity of LHS 228 could however be between -1.0 and -0.5 ; this would increase the age range from $0.11-0.25$ to 0.25-10.0 Gyr making it more consistent with the expectation for a subdwarf. LHS 360 would fit a lower metallicity model but that requires a very young age.

\section{Conclusions}

We have measured the parallaxes of 27 cool dwarf stars and compared them to a set of models. A number of high proper motion objects, planetary nebulae and cataclysmic variables observed in the TOPP remained unpublished; we plan to release these in the near future. There are no plans to begin any more large programs with the Torino telescope in the near future. If the Gaia mission, in which Torino is heavily involved, is successful then there will no longer be any need for parallaxes from $1 \mathrm{~m}$-class telescopes.

The majority of ground-based parallax programs are now concentrated on the newly discovered brown dwarfs. These have very red colours and will be beyond the magnitude limit of Gaia, requiring very red or infrared observations on $4 \mathrm{~m}$ class telescopes. The denser and more precise reference frame from Gaia will enable us to improve our reduction strategy and the TOPP observations remain a development test bed for this. Current activity is focused on the calculation of the relative-to-absolute parallax correction, treatment of binaries, and reference frame selection.

Acknowledgements. We would like to thank the referee, Dr S. K. Leggett, who's comments significantly improved the quality of this paper.

H.R.A.J. and R.L.S. acknowledge the support of Royal Society International Joint Project 2007/R3. B.B., M.G.L. and R.L.S. acknowledge the support of INAF through the PRIN 2007 grant n. CRA 1.06.10.04.

This research has made use of: the SIMBAD database operated at CDS France, the Second Guide Star Catalog developed as a collaboration between the Space Telescope Science Institute and the Osservatorio Astronomico di Torino, and, the Two Micron All Sky Survey which is a joint project of the University of Massachusetts and the Infrared Processing and Analysis Center/California Institute of Technology.

\section{References}

Balega, I., Balega, Y. Y., Maksimov, A. F., et al. 2004, A\&A, 422, 627 Bessell, M. S. 1990, A\&AS, 83, 357 Bidelman, W. P. 1985, ApJS, 59, 197

Bucciarelli, B., García Yus, J., Casalegno, R., et al. 2001, A\&A, 368, 335 Carpenter, J. M. 2001, AJ, 121, 2851

Costa, E., Méndez, R. A., Jao, W.-C., et al. 2005, AJ, 130, 337

Costa, E., Méndez, R. A., Jao, W.-C., et al. 2006, AJ, 132, 1234

Dahn, C. C., Harrington, R. S., Riepe, B. Y., et al. 1982, AJ, 87, 419

Dahn, C. C., Harris, H. C., Vrba, F. J., et al. 2002, AJ, 124, 1170

Dawson, P. C., \& De Robertis, M. M. 1989, AJ, 98, 1472

Dawson, P. C., \& Forbes, D. 1989, PASP, 101, 614

Dehnen, W., \& Binney, J. J. 1998, MNRAS, 298, 387

Docobo, J. A., Tamazian, V. S., Balega, Y. Y., \& Melikian, N. D. 2006, AJ, 132, 994

Fleming, T. A., Giampapa, M. S., Schmitt, J. H. M. M., \& Bookbinder, J. A. 1993, ApJ, 410, 387

Froeschle, M., Kovalevsky, J., \& Mignard, F. 1985, Correction of veiling glare in HIPPARCOS observations, Tech. rep.

Gizis, J. E., Reid, I. N., \& Hawley, S. L. 2002, AJ, 123, 3356

Gliese, W., \& Jahreiß, H. 1991, On: The Astronomical Data Center CDROM: Selected Astronomical Catalogs, Vol. I, ed. S. G. e. L.E. Brotzmann (Greenbelt, MD: NASA/Astronomical Data Center, Goddard Space Flight Center)

Harrington, R. S., Dahn, C. C., Kallarakal, V. V., et al. 1993, AJ, 105, 1571 Harrington, R. S., Kallarakal, V. V., Christy, J. W., et al. 1985, AJ, 90, 123

Hauschildt, P. H., Allard, F., Ferguson, J., Baron, E., \& Alexander, D. R. 1999, ApJ, 525, 871

Heintz, W. D. 1994, AJ, 108, 2338

Henry, T. J., Subasavage, J. P., Brown, M. A., et al. 2004, AJ, 128, 2460

Holmberg, J., Nordström, B., \& Andersen, J. 2009, A\&A, 501, 941

Ianna, P. A., Rohde, J. R., \& McCarthy, Jr., D. W. 1988, AJ, 95, 1226

Jao, W., Henry, T. J., Beaulieu, T. D., \& Subasavage, J. P. 2008, AJ, 136, 840

Jao, W.-C., Henry, T. J., Subasavage, J. P., et al. 2005, AJ, 129, 1954

Johnson, J. A., \& Apps, K. 2009, ApJ, 699, 933

Jones, H. R. L., \& Tsuji, T. 1997, ApJ, 480, L39 
A\&A 514, A84 (2010)

Jurić, M., Ivezić, Z., Brooks, A., et al. 2008, APJ, 673, 864

Kilkenny, D., Koen, C., van Wyk, F., Marang, F., \& Cooper, D. 2007, MNRAS, 380, 1261

Kirkpatrick, J. D., Henry, T. J., \& Simons, D. A. 1995, AJ, 109, 797

Lanteri, L. 1990, Robin Software, Tech. Rep. 16, Osservatorio Astronomico di Torino

Lasker, B. M., Lattanzi, M. G., McLean, B. J., et al. 2008, AJ, 136, 735

Leggett, S. K. 1992, APJS, 82, 351

Lépine, S., Rich, R. M., \& Shara, M. M. 2007, ApJ, 669, 1235

Lyubchik, Y., Jones, H. R. A., Pavlenko, Y. V., et al. 2007, A\&A, 473, 257

Martín, E. L., Basri, G., \& Zapatero Osorio, M. R. 1999, AJ, 118, 1005

Mathioudakis, M., \& Doyle, J. G. 1991, A\&A, 244, 409

McCarthy, Jr., D. W., Henry, T. J., Fleming, T. A., et al. 1988, ApJ, 333, 943

Mendez, R. A., \& van Altena, W. F. 1996, AJ, 112, 655

Monet, D. G., Dahn, C. C., Vrba, F. J., et al. 1992, AJ, 103, 638

Perryman, M. A. C., Lindegren, L., Kovalevsky, J., et al. 1997, A\&A, 323, L49

Shkolnik, E., Liu, M. C., \& Reid, I. N. 2009, ApJ, 699, 649
Smart, R. L., Bucciarelli, B., Lattanzi, M. G., Massone, G., \& Chiumiento, G. 1999, A\&A, 348, 653

Smart, R. L., Lattanzi, M. G., Bucciarelli, B., et al. 2003, A\&A, 404, 317

Smart, R. L., Lattanzi, M. G., \& Drimmel, R. 1997, in Wide-field spectroscopy, ed. E. Kontizas, M. Kontizas, D. H. Morgan, \& G. P. Vettolani, Astrophysics and Space Science Library, 212, 195

Smart, R. L., Lattanzi, M. G., Jahreis, H., Bucciarelli, B., \& Massone, G. 2007, A\&A, 464, 787

Stearns, C. L. 1959, AJ, 64, 269

Strand, K. A. 1966, Vistas Astron., 8, 9

Torres, G. 2007, ApJ, 671, L65

van Altena, W. F., Lee, J. T., \& Hoffleit, E. D. 2001, VizieR Online Data Catalog, 1238,0

van Maanen, A. 1944, ApJ, 100, 55

Weis, E. W. 1996, AJ, 112, 2300

Weistrop, D. 1979, PASP, 91, 193

Zuckerman, B., \& Song, I. 2004, ARA\&A, 42, 685 